\title{
Science and technical progress at the Palomar Testbed Interferometer
}

\section{Rachel L. Akeson}

Rachel L. Akeson, "Science and technical progress at the Palomar Testbed Interferometer," Proc. SPIE 7013, Optical and Infrared Interferometry, 701307 (28 July 2008); doi: $10.1117 / 12.787429$

Event: SPIE Astronomical Telescopes + Instrumentation, 2008, Marseille, France 


\title{
Science and technical progress at the Palomar Testbed Interferometer
}

\author{
Rachel L. Akeson ${ }^{* a}$ and the PTI Collaboration \\ ${ }^{a}$ Michelson Science Center, California Institute of Technology, Pasadena, CA, USA
}

\begin{abstract}
The Palomar Testbed Interferometer (PTI) is a long-baseline, near-infrared interferometer located on Palomar Mountain. PTI has been in operation since 1997 and is used for extensive science observing as well as technical development. I will review the recent progress at PTI covering both science and instrumentation work. The science topics include fundamental stellar properties, novae, and an evaluation of all calibrator observations. The technical work includes the narrow-angle phase scanning mode and installation of the integrated optics combiner IONIC.
\end{abstract}

Keywords: long-baseline interferometry, data archive

\section{INTRODUCTION}

The Palomar Testbed Interferometer (PTI) is a long-baseline, fringe-tracking interferometer which operates in the nearinfrared bands and is located at the Palomar Observatory, Palomar Mountain, California. PTI was constructed by the Jet Propulsion Laboratory, Caltech, with funding from the National Aeronautics and Space Administration as a technical testbed for the Keck Interferometer, specifically for demonstration of dual-star, narrow-angle astrometry and phase referencing between two adjacent stars. PTI obtained first fringes in 1995 and has been in routine science operations since 1997. PTI science contributions were last reviewed in 2006 by Akeson ${ }^{1}$ at the previous meeting of this conference and this paper will concentrate on results since then.

A full technical description of PTI can be found in Colavita et $\mathrm{al}^{2}$. PTI currently has three operational stations, which can be combined pair-wise into three baselines, two $\sim 85$ meters and one $\sim 110$ meters in length, resulting in typical fringe spacings of 4 to 5 milliarcsecs at K-band (2.2 microns). PTI has a high science data throughput due to the efficient fringe tracking and the highly automated nighttime operations which are run in a queue-scheduled mode.

\subsection{Current Operations}

PTI currently operates in three modes: 1) visibility amplitude mode using fringe tracking in $\mathrm{H}$ (1.6 microns) or K bands, 2) fringe scanning in $\mathrm{K}$ band, and 3 ) visibility amplitudes using fringe scanning at $\mathrm{H}$ band with the IONIC combiner. The K-band fringe scanning mode is described by Lane and Mutterspaugh ${ }^{3}$ and is used by the PHASES (Palomar Highprecision AStrometric Exoplanet Search; S. Kulkarni, PI) Key Project. The visibility amplitude observations encompass a range of smaller programs, with example results discussed in Section 2. The highest sensitivity mode is $\mathrm{K}$ band fringe tracking where good science data are possible down to $\mathrm{K}=6$.

PTI is supported by the PTI collaboration (http://msc.caltech.edu/missions/Palomar) and administered by the Michelson Science Center (MSC) for the collaboration and the JPL/Caltech community, although proposals from outside these communities are accepted. Funding for operations is provided by contributions from the MSC, the Space Interferometry Mission (SIM-PlanetQuest), the PHASES project, and the Infrared Processing and Analysis Center (IPAC). More information on available PTI support from the MSC can be found at http://msc.caltech.edu/software/PTISupport. PTI is fully funded for operations through 2008.

\subsection{Technical Developments}

No major changes have been made to the fringe tracking mode in the last several years. Operations and technical improvements to the fringe scanning mode are described as part of the PHASES Key Science Project results (section 2.2). The major development effort at PTI in the last two years has been the installation and operation of the integrated

\footnotetext{
${ }^{*}$ rla@ipac.caltech.edu
}

Optical and Infrared Interferometry, edited by Markus Schöller, William C. Danchi, Françoise Delplancke Proc. of SPIE Vol. 7013, 701307, (2008) · 0277-786X/08/\$18 - doi: 10.1117/12.787429 
optics component of the Integrated Optics Near-infrared Interferometric Camera (IONIC) beam combiner (described in detail by Berger et $\mathrm{al}^{4}$ ) which was formerly used at the Infrared Optical Telescope Array (IOTA). H-band light is extracted using dichroics and fold mirrors placed between the PTI beam-reducing telescopes and the short delay lines. The $20 \mathrm{~mm}$ beams at PTI are injected into two of the three input fibers of IONIC using gold-coated off-axis paraboloids of $90 \mathrm{~mm}$ effective focal-length. The post-combination optics and detector are identical to those used at IOTA, and the detector is read-out with the same electronics that operates the PTI secondary table, as used by PHASES.

Originally, this beam-combiner was intended to provide a path to three-beam combination at PTI, but is currently being used in a two-beam mode with photometric outputs. Of the six outputs of the IONIC combiner two are differenced to provide a fringe signal, and the other four are used in two pairs of two outputs to provide photometric outputs. The use of the IONIC beam combiner at H-band while fringe-tracking at K-band maintains the fringe-tracking sensitivity of the PTI primary table while enabling spatially-filtered well-calibrated observations in H-band. Once fringes are acquired, IONIC data is recorded in a fringe-scanning mode, with a 40 micron scan length in optical-path-difference. The scanning provided by scanning the short and long delay lines at PTI in antiphase, and the $1 \lambda$ "stroke" for primary table fringetracking is provided by the short delay line. The IONIC combiner therefore sees the phase corrections made by the primary table and the 40 micron scan of the long delay line, while the primary table does not see this scan, and only sees the $1 \lambda$ "stroke" used for phase modulation. The installation and test of the IONIC mode at PTI has been led by M. Ireland and this mode is fully operational for the 2008 observing season. The use of IONIC at PTI was made possible by W. Traub and J.P. Berger.

\section{RECENT SCIENCE RESULTS}

PTI has 12 published peer-reviewed science papers between 2006 and the present time. The results of these papers are briefly summarized below and the papers are given in the reference section. The complete PTI science publication list is available at http://msc.caltech.edu/missions/Palomar/publications.html.

\subsection{Fundamental stellar properties}

As with many of the smaller aperture interferometers, PTI spends a substantial portion of its observing time on measurements designed to precisely determine fundamental stellar parameters and therefore to test models of stellar structure and evolution.

Binary stars: Interferometry in conjunction with radial velocity measurements can be used to solve for the full physical orbit and distance in binary systems. Then in combination with the flux ratios, the mass, luminosity, and radius can be measured, in some cases to better than $1 \%$.

Boden et al. ${ }^{5}$ reported on spectroscopic and interferometric observations of the high-proper motion double-lined binary system HD 9939, which has an orbital period of approximately 25 days. By combining radial-velocity and visibility measurements they estimated the system physical orbit and derived dynamical masses for the components of $\mathrm{M}_{\mathrm{A}}=1.072$ $\pm 0.014 \mathrm{M}_{\odot}$ and $\mathrm{M}_{\mathrm{B}}=0.8383 \pm 0.0081 \mathrm{M}_{\odot}$; fractional errors of $1.3 \%$ and $1.0 \%$, respectively. A system distance of $42.23 \pm 0.21 \mathrm{pc}$ was determined, which corresponds to an orbital parallax of $\pi_{\mathrm{orb}}=23.68 \pm 0.12$ milliarcsec. The system distance and the estimated brightness difference between the stars in $\mathrm{V}, \mathrm{H}$, and $\mathrm{K}$ bands yielded component absolute magnitudes in these bands. By spectroscopic analysis and spectral energy distribution modeling they estimated the component effective temperatures and luminosities as $\mathrm{T}_{\text {eff }}(\mathrm{A})=5050 \pm 100 \mathrm{~K}, \mathrm{~T}_{\text {eff }}(\mathrm{B})=4950 \pm 200 \mathrm{~K}, \mathrm{~L}_{\mathrm{A}}=2.451 \pm$ $0.041 \mathrm{~L}_{\odot}$, and $\mathrm{L}_{\mathrm{B}}=0.424 \pm 0.023 \mathrm{~L}_{\odot}$. Both the spectral analysis and comparison with stellar models suggest that $\mathrm{HD}$ 9939 has elemental abundances near solar values. Further, comparison with stellar models suggests the HD 9939 primary has evolved off the main sequence and appears to be traversing the Hertzsprung gap as it approaches the red giant phase of its evolution. These measurements of the primary properties provide new empirical constraints on stellar models during this particularly dynamic evolutionary phase. As HD 9939 is currently in a relatively short-lived evolutionary state, the system age can be estimated as $9.12 \pm 0.25$ Gyr. In turn, the age and abundance of the system place a potentially interesting constraint on star formation in the galactic disk. 
Stellar radii: van Belle et al. ${ }^{6}$ report on direct angular size measurements of the G0 IV subgiant $\eta$ Boo. They found a limb-darkened angular size of $\theta_{\mathrm{LD}}=2.1894+0.0055-0.0140$ mas, which gives a linear radius of $\mathrm{R}=2.672 \pm 0.028 \mathrm{R} \odot$. They computed a bolometric flux estimate of $\mathrm{F}=(22.1 \pm 0.28) \times 10^{-7} \mathrm{ergs} / \mathrm{cm}^{2} / \mathrm{s}$, an effective temperature of $\mathrm{T}_{\text {eff }}=6100 \pm 28 \mathrm{~K}$, and a luminosity of $\mathrm{L}=8.89 \pm 0.16 \mathrm{~L} \odot$. In conjunction with the mass estimate from the MOST investigation ${ }^{7}$, a surface gravity of $\log g=3.817 \pm 0.016\left[\mathrm{~cm} / \mathrm{s}^{2}\right]$ is established for $\eta$ Boo. The measurement of the surface gravity, independent of spectroscopy, is a significant demonstration of the astrophysical investigative value of combining high spatial resolution interferometry with high temporal resolution photometry and this value has a stated uncertainty an order of magnitude greater than previous measures.

\subsection{The PHASES exoplanet search project}

The Palomar High-precision Astrometric Search for Exoplanet Systems (PHASES) Key Science program uses a fringescanning technique ${ }^{3}$ to obtain high-precision (10-20 micro-arcsec) astrometry of close stellar pairs. Muterspaugh et al. ${ }^{8}$ describe these observations which provide precise visual orbits of the binaries and allow detection of tertiary components orbiting either the primary or secondary by way of the reflex motion of the subsystem center of light. PHASES measures the separation vectors of bright binaries that are not resolved by PTI's telescopes but are overresolved by the interferometer itself. Phase referencing is used to maintain coherence so that the full resolving power of the interferometer is applied to the astrometric measurement. The current limiting magnitude in phasereferencing mode is $\mathrm{K} \sim 4.5$ for equal-magnitude binaries. Additional companions to the primary and/or secondary stars will produce perturbations to the binary Keplerian orbit. Distant companions simultaneously orbiting both visible components will cause only very small perturbations to the observed separation vector (the differential gravitational pull is small); the PHASES observations are not sensitive to these circumbinary planets, also called "P-type" or planetarytype planets. However, companions orbiting just one star of the binary can cause noticeable perturbations to the separation vectors. PHASES is a search for these planetary systems, also called "S-type" or satellite-type planets. The primary goal of the PHASES program is to find and characterize giant planets in close binary systems (semimajor axis $<50 \mathrm{AU})$.

During the development of this mode potential sources of systematic instrumental errors were identified, and efforts made to correct them ${ }^{8}$. The first source is color dispersion within the interferometer, which has two effects. The optical path equalization at PTI is done with delay lines in air, which has a wavelength-dependent index of refraction. The fringe packets of astrophysical sources are therefore dispersed by an amount that depends on the air path difference, changing the shape and overall location of the fringe packets. If the two stars have different colors, each is dispersed by a slightly different amount, and their apparent separation will be biased. In addition, if there is dispersion perpendicular to the light beam or diffraction, the color of the star's light falling on the image plane detector pixel can vary with sky position. In the presence of longitudinal dispersion, the dependence of these color shifts on location within the pixel can also introduce astrometric systematics. A longitudinal dispersion compensator was installed to eliminate both systematics. A second source of potential error is drift in the "astrometric baseline" due to drifts in optical alignment, which was addressed by introduction of an automatic alignment system.

Muterspaugh et al. ${ }^{8}$ examined the characteristics of the noise in these fringe-scanning observations and described an approach to differentiate between contributions from the noise and the potential companions by recognizing that an astrometric perturbation will appear as a Keplerian wobble, whereas instrumental terms will be random. They describe an algorithm for determining the confidence levels of such a signal in the presence of instrumental scatter and apply this algorithm to 8 multiple systems. The PHASES observations exclude tertiary companions with masses as small as a few Jupiter masses in several binary systems (e.g Figure 1). The phase-space searching algorithm correctly identifies the tertiary in two previously studied triple star systems and found the correct periods for the short period pairs. 


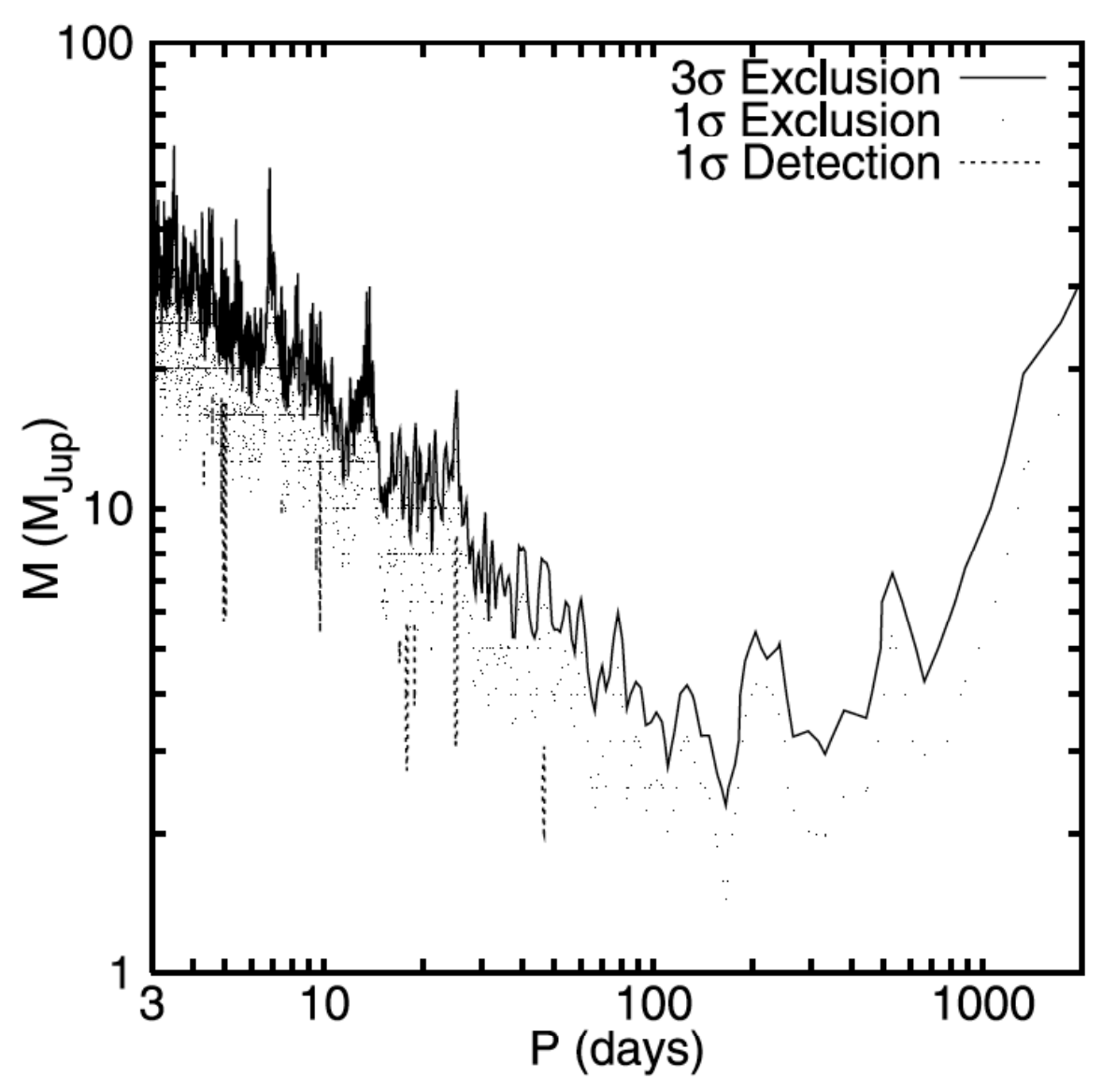

Figure 1: The mass-period phase space excluded by the PHASES data on 13 Pegasi. Objects as small as $2 \mathrm{M}_{\text {Jup }}$ are ruled out for some periods. Figure from Muterspaugh et $\mathrm{al}^{8}$.

The PHASES group have also used this astrometric technique to study in detail the stellar and orbital properties of several triple and quadruple systems including, $\kappa \mathrm{Peg}^{9}$, V819 Herculis ${ }^{10}, 88 \mathrm{Tau} \mathrm{A}^{11}$ and $\mu$ Orionis ${ }^{12}$. The data for the quadruple system $\mu$ Ori show both the motion of the long period binary orbit and the short period perturbations caused by each of the components in the long period system being themselves binaries. Muterspaugh et $\mathrm{al}^{12} \mathrm{determined} \mathrm{the}^{2}$ orientations of the long period binary and short period subsystems (Figure 2). Recent theoretical work predicts the distribution of relative inclinations between inner and outer orbits of hierarchical systems to peak near 40 and 140 degrees. The degree of coplanarity of this complex system is determined, and the angle between the planes of the A-B and $\mathrm{Aa}-\mathrm{Ab}$ orbits is found to be $136.7 \pm 8.3$ degrees, near the predicted distribution peak at 140 degrees. 

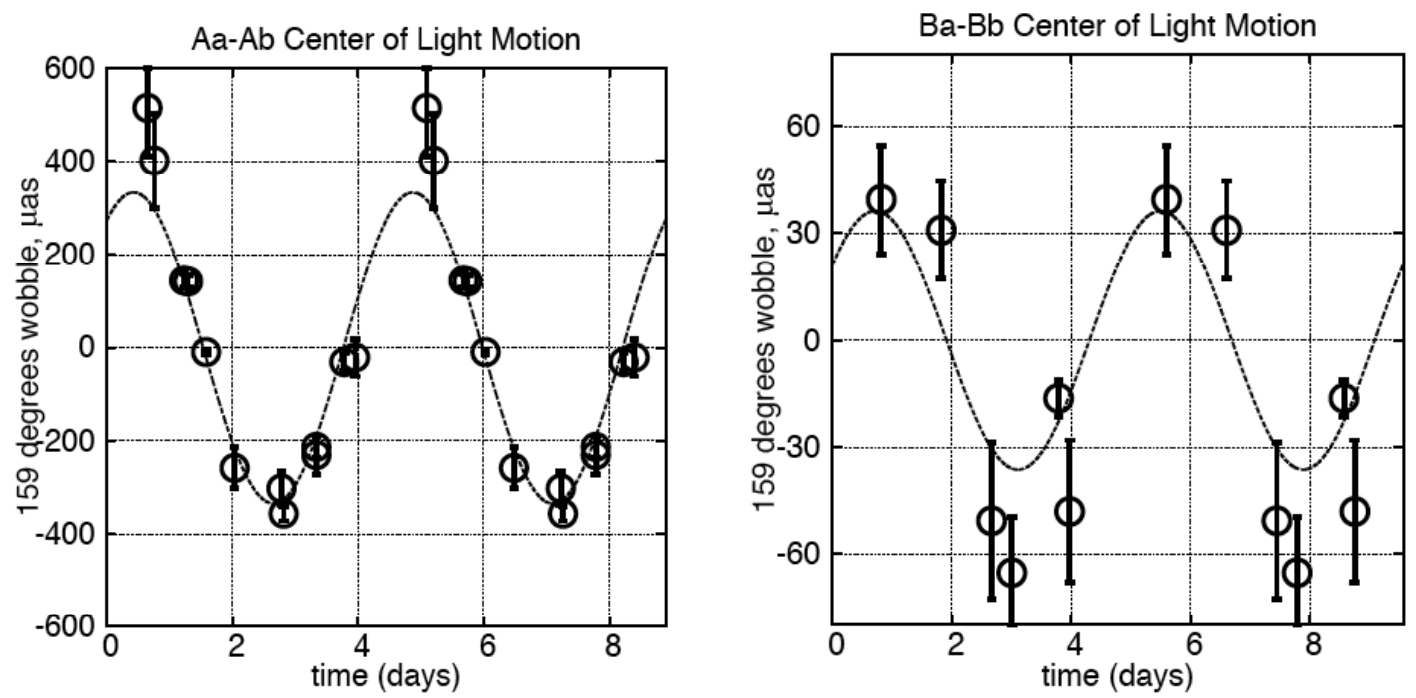

Figure 2: The astrometric orbits of $\mu$ Ori $\mathrm{Aa}-\mathrm{Ab}$ and $\mathrm{Ba}-\mathrm{Bb}$, phase-wrapped about their respective orbital periods. Phase zero is at the epoch of periastron passage, and each plot is repeated for two cycles (and each measurement is plotted twice) to allow for continuity at all parts of the graph. In both cases, the motions of the A-B system and of the other subsystem have been removed. The projection axis shown for each is 159 degrees East of North. Figure from Muterspaugh et $\mathrm{al}^{12}$.

\subsection{Evolved stars}

PTI has also dedicated substantial observing time to the study of evolved stars. van Belle et al. ${ }^{13}$ have studied the effective temperature as a function of V-K color for supergiants (luminosity class I and II) and found that they appear to follow the same function as giants ${ }^{14}$ for V-K values less than 5. Departure from this relation (Figure 3) indicates the onset of mass loss. 


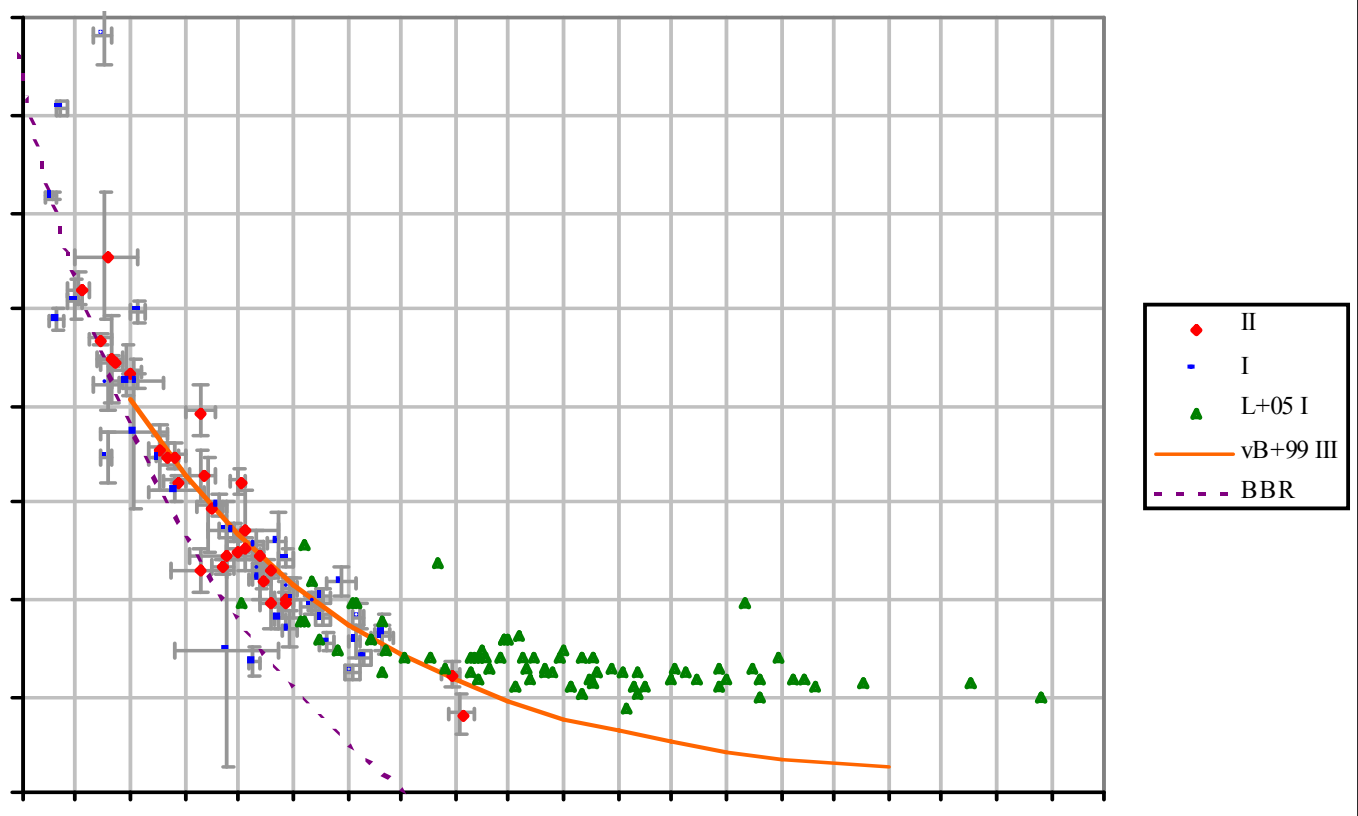

Figure 3: The derived effective temperature as a function of V-K color for luminosity class I (points) and II (diamonds) stars. Effective temperature determinations from Levesque et al. ${ }^{15}$ using spectroscopy are shown as triangles. The giant star relation derived by van Belle et al. ${ }^{14}$ is shown as a red line. Figure provided by G. van Belle.

\subsection{Novae}

One of the long-standing target of opportunity programs at PTI has been following nearby novae when they are sufficiently bright to meet the sensitivity limits.

V1663 Aql: Lane et al. ${ }^{16}$ resolved the classical nova V1663 Aql with observations covering from $\sim 5$ to 18 days after peak brightness. They directly measured the shape and size of the fireball, which was found to be asymmetric. They also measured an expansion rate of $0.21+/-0.03 \mathrm{mas} /$ day and assuming a linear expansion model, inferred that the initial outburst occured approximately 4 days prior to peak brightness.

RS Oph: Two separate groups used PTI data on the recurrent nova, RS Oph, which had an outburst on February 12, 2006, and came to different conclusions as to the morphology of the near-infrared emitting component. Monnier et al. ${ }^{17}$ used data from the IOTA, Keck, and PTI Interferometers at multiple epochs. They found that the characteristic size of $\sim 3$ mas hardly changed over the first 60 days of the outburst, ruling out currently popular models whereby the near-infrared emission arises from hot gas in the expanding shock. The emission was also found to be significantly asymmetric, supported primarily by the nonzero closure phases detected by IOTA. Lane et al. ${ }^{18}$ used additional data taken at PTI and concluded that the near-IR source initially expands to a size of approximately 5 milli-arcseconds. However, beginning around day 10 the IR source appears to begin to shrink, reaching approximately 2 milli-arcseconds by day 100 .

\subsection{Young stellar objects}

PTI has been quite influential in the study of circumstellar disks around young stellar objects, as it made the first observations of both an $\mathrm{FU}_{\mathrm{Ori}}{ }^{19}$ and T Tauri object ${ }^{20}$. Eisner et al. ${ }^{21}$ used the spectrally dispersed $\mathrm{K}$ band data to constrain the temperature profiles of sub-AU-sized regions of $11 \mathrm{Herbig} \mathrm{Ae} / \mathrm{Be}$ sources (intermediate mass young stars). They found that a single-temperature ring does not reproduce the data well (Figure 4). Comparison of models with 
broadband spectral energy distributions led them to suggest separate gas and dust components with different temperatures and spatial distributions.

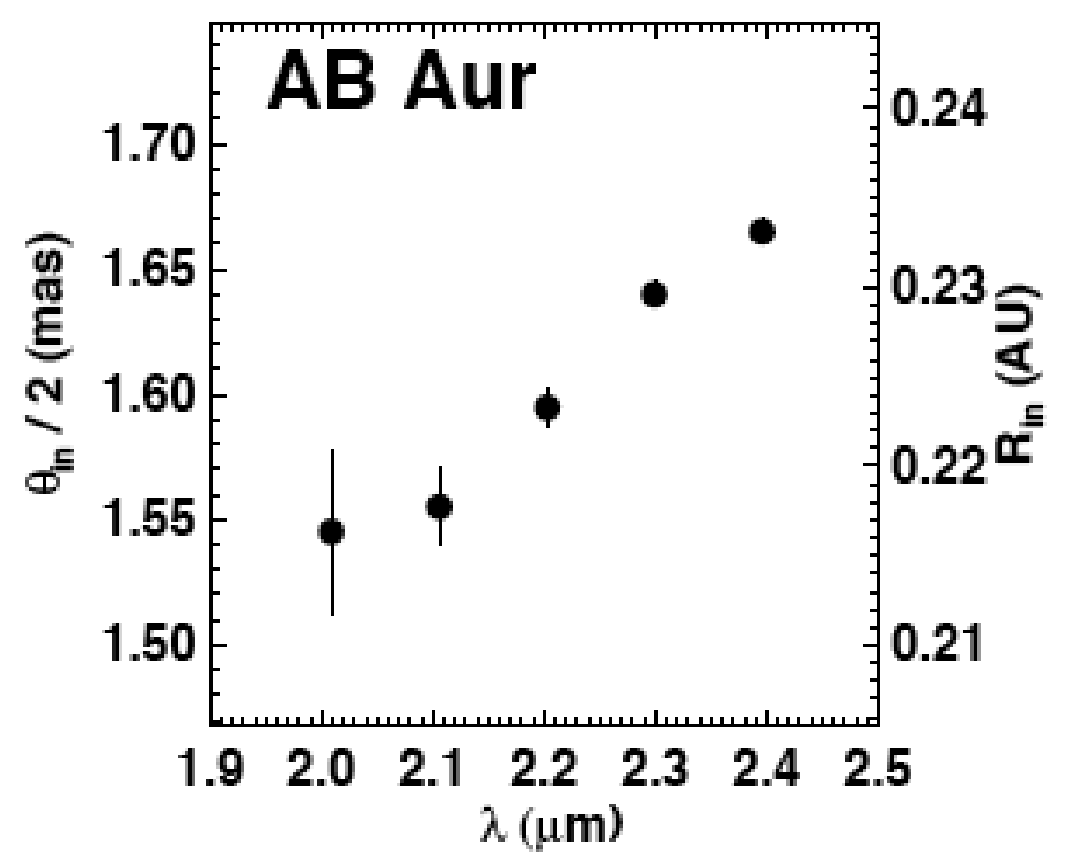

Figure 4: The measured angular radius as a function of wavelength for the young intermediate mass star, AB Aur. Figure from Eisner et al. ${ }^{21}$

\subsection{PTI Calibrator Catalog}

van Belle et al. ${ }^{22}$ examined the archive of PTI observations between 1998 and 2005 for objects appropriate for calibration, i.e. stars that are predictably point-like and single. Approximately 1,400 nights of data on 1,800 objects were examined for this investigation. They compared those observations to an intensively studied object that is a suitable calibrator, HD217014, and statistically compared each candidate calibrator to that. Their investigation was designed to test the hypothesis that the frequency distribution of visibility data associated with calibrator stars differs from noncalibrator stars such as binary stars. Spectroscopic binaries resolved by PTI, objects known to be unsuitable for calibrator use, were similarly tested to establish detection limits. They found more than 350 observed stars suitable for use as calibrators (with an additional 140 being rejected), corresponding to $>95 \%$ sky coverage for PTI. This approach rigorously establishes calibration sources through a traceable, empirical methodology, leveraging the predictions of spectral energy distribution modeling but also verifying it with on-sky observations. 


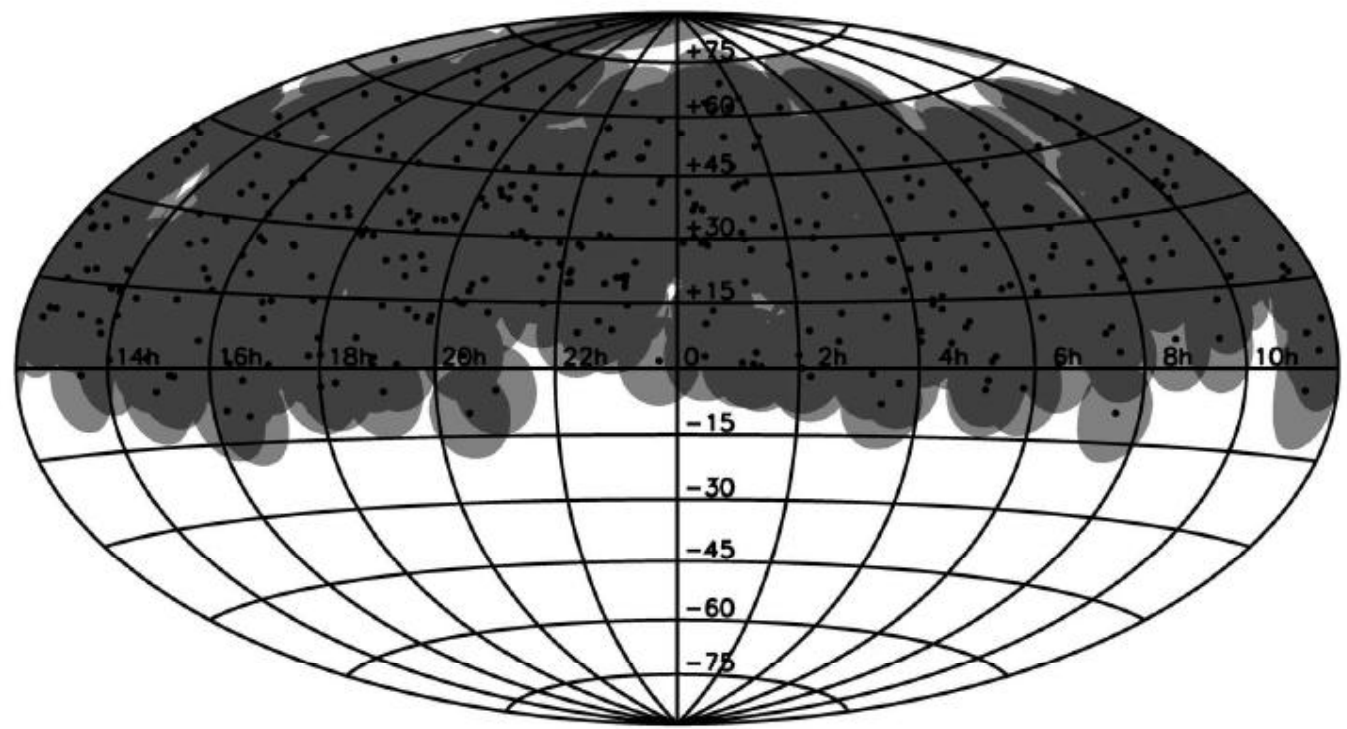

Figure 5: An Aitoff projection of the sky coverage from the PTI calibrator catalog. The light grey circles represent 10 degrees cones and the darker regions are where one or more cones overlap. For PTI's nominal declination range of 5 to 55 degrees, this represents $>96 \%$ sky coverage. Figure from van Belle et al. ${ }^{22}$

\section{PTI DATA ARCHIVE}

As introduced in the last proceedings of this conference, PTI visibility amplitude data is now available in a public archive at https://mscweb.ipac.caltech.edu/mscdat-pti/secure/main.jsp. Data are available to public users 18 months after the observation date. The archive now contains data from over 1350 nights and over 1300 sources. In addition to supporting the archive, the MSC provides data reduction packages and contact science assistance. For more details, see http://msc.caltech.edu/software/PTISupport/. 


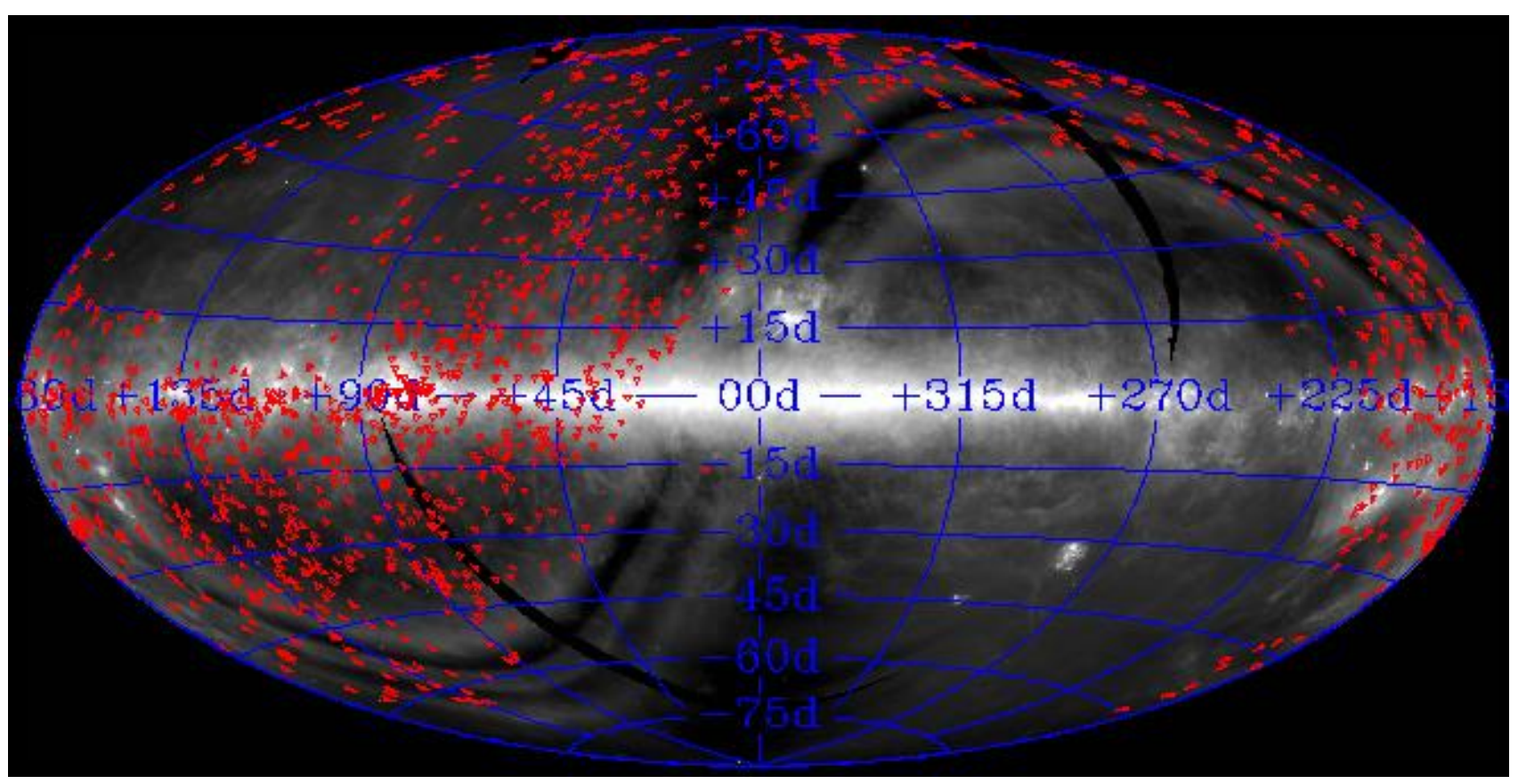

Figure 6: The distribution of sources in the PTI data archive plotted against the IRAS galactic emission.

Acknowledgements: PTI was designed for an operational lifetime of 3 years, but has produced scientifically relevant results for over 10 years. This is the result of the work and dedication of many people, in particular the project architect Mark Colavita and our excellent observers, Jean Mueller and Kevin Rykoski. In the last two years, the following have provided particular operations and development support to PTI: A. Boden, E. Hovland, M. Ireland, B. Lane, M. Muterspaugh, B. Thompson and G. Vasisht. Thanks to G. van Belle and M. Ireland for providing material for this review.

\section{REFERENCES}

[1] Akeson, R.L., "Recent progress at the Palomar Testbed Interferometer", SPIE, 6268, 14 (2006)

[2] Colavita, M. M., et al., "The Palomar Testbed Interferometer", Astrophysical Journal, 510, 505 (1999)

[3] Lane, B.F. and Muterspaugh, M.W., "Differential Astrometry of Subarcsecond Scale Binaries at the Palomar Testbed Interferometer", Astrophysical Journal, 601, 1129 (2004)

[4] Berger, J.P. et al," An integrated-optics 3-way beam combiner for IOTA",SPIE, 4838, 1099 (2003)

[5] Boden, A.F., Torres, G., and Latham, D.W., "A Physical Orbit for the High Proper Motion Binary HD 9939", Astrophysical Journal, 644, 1193 (2006)

[6] van Belle, G.T., Ciardi, D.R., \& Boden, A.F, "Measurement of the Surface Gravity of $\eta$ Boo", Astrophysical Journal, 657, 1058 (2007)

[7] Guenther, D.B., et al," Stellar Model Analysis of the Oscillation Spectrum of $\eta$ Bootis Obtained from MOST", Astrophysical Journal, 635, 547 (2005)

[8] Muterspaugh, M.W. et al., "Limits to Tertiary Astrometric Companions in Binary Systems", Astrophysical Journal, 653, 1469 (2006)

[9] Muterspaugh, M.W. et al., "PHASES Differential Astrometry and Iodine Cell Radial Velocities of the $\kappa$ Pegasi Triple Star System”, Astrophysical Journal, 636, 1020 (2006) 
[10] Muterspaugh, M.W. et al., "PHASES differential astrometry and the mutual inclination of the V819 Herculis triple star system", Astronomy \& Astrophysics, 446, 723 (2006)

[11] Lane, B. F., et al, "The Orbits of the Quadruple Star System 88 Tauri A from PHASES Differential Astrometry and Radial Velocity”, Astrophysical Journal, 669, 1209 (2007)

[12] Muterspaugh, M.W. et al., "Masses, Luminosities, and Orbital Coplanarities of the $\mu$ Orionis Quadruple Star System from PHASES Differential Astrometry”, Astronomical Journal, 135, 766 (2008)

[13] van Belle, G.T, et al, in preparation

[14] van Belle, G.T. et al., "Radii and Effective Temperatures for G, K, and M Giants and Supergiants", Astronomical Journal, 117, 521 (1999)

[15] Levesque, E.M., et al., "The Effective Temperature Scale of Galactic Red Supergiants: Cool, but Not As Cool As We Thought", Astrophysical Journal, 628, 973 (2005)

[16] Lane, B. F, et al, "Interferometric Observations of V1663 Aquilae (Nova Aql 2005)", Astrophysical Journal, 669, 1150 (2007)

[17] Monnier, J. D., et al, "No Expanding Fireball: Resolving the Recurrent Nova RS Ophiuchi with Infrared Interferometry", Astrophysical Journal, 647, 127 (2006)

[18] Lane, B. F., et al., "Interferometric Observations of RS Ophiuchi and the Origin of the Near-IR Emission", Astrophysical Journal, 658 , 520 (2006)

[19] Malbet, F., et al.," FU Orionis Resolved by Infrared Long-Baseline Interferometry at a 2 AU Scale”, Astrophysical Journal, 507, 149 (1999)

[20] Akeson, R.L. et al., "Infrared Interferometric Observations of Young Stellar Objects", Astrophysical Journal, 543, $131(2000)$

[21] Eisner, J. A., et al, "Spectrally Dispersed K-Band Interferometric Observations of Herbig Ae/Be Sources: Inner Disk Temperature Profiles", Astrophysical Journal, 657, 347 (2007)

[22] van Belle, G.T., et al, "The Palomar Testbed Interferometer Calibrator Catalog”, Astrophysical Journal Supplement Series., 176, $276(2008)$ 